\title{
Parylene photonics: a flexible, broadband optical waveguide platform with integrated micromirrors for biointerfaces
}

\author{
Jay W. Reddy ${ }^{1}$, Maya Lassiter ${ }^{1}$ and Maysamreza Chamanzar ${ }^{1}$
}

\begin{abstract}
Targeted light delivery into biological tissue is needed in applications such as optogenetic stimulation of the brain and in vivo functional or structural imaging of tissue. These applications require very compact, soft, and flexible implants that minimize damage to the tissue. Here, we demonstrate a novel implantable photonic platform based on a highdensity, flexible array of ultracompact $(30 \mu \mathrm{m} \times 5 \mu \mathrm{m})$, low-loss $(3.2 \mathrm{~dB} / \mathrm{cm}$ at $\lambda=680 \mathrm{~nm}, 4.1 \mathrm{~dB} / \mathrm{cm}$ at $\lambda=633 \mathrm{~nm}$, $4.9 \mathrm{~dB} / \mathrm{cm}$ at $\lambda=532 \mathrm{~nm}, 6.1 \mathrm{~dB} / \mathrm{cm}$ at $\lambda=450 \mathrm{~nm}$ ) optical waveguides composed of biocompatible polymers Parylene $C$ and polydimethylsiloxane (PDMS). This photonic platform features unique embedded input/output micromirrors that redirect light from the waveguides perpendicularly to the surface of the array for localized, patterned illumination in tissue. This architecture enables the design of a fully flexible, compact integrated photonic system for applications such as in vivo chronic optogenetic stimulation of brain activity.
\end{abstract}

\section{Introduction}

From biological science to clinical practice, optical methods for imaging ${ }^{1-3}$ and manipulation ${ }^{4-9}$ of tissue are the gold standard for noninvasive interaction. However, the scattering and absorption of light in tissue pose fundamental limitations to the achievable resolution and depth of penetration. Depending on the type of tissue and wavelength of light, noninvasive optical microscopy techniques are typically limited to the superficial layers of tissue due to scattering and absorption of light, especially in the visible range of the optical spectrum ${ }^{10}$. Multiphoton techniques, which utilize simultaneous absorption of longer wavelength photons to activate visible-range optical agents, achieve deeper penetration into tissue due to reduced attenuation of near-infrared and infrared light. Even when using advanced multiphoton techniques, optical access is still limited to a couple of millimeters deep into the tissue ${ }^{11}$. In the context of brain imaging and manipulation, accessing deeper regions is required to

\footnotetext{
Correspondence: Maysamreza Chamanzar (mchamanzar@cmu.edu)

${ }^{1}$ Department of Electrical and Computer Engineering, Carnegie Mellon

University, Pittsburgh, PA, USA
}

study the neural mechanisms of disorders such as Parkinson's disease that involve malfunction of circuits in deep structures, namely, the basal ganglia nuclei. The issue of penetration depth is even more limiting for studying the large brains of nonhuman primates and humans. Implantable devices, which enable targeted light delivery deep into tissue, are therefore needed to advance scientific understanding of biological mechanisms and to aid clinical intervention.

Optical imaging and manipulation in free-roaming animal subjects require miniaturized technologies that are much smaller than traditional bulky microscopes. Recently demonstrated miniaturized microscopes and miniscopes, powered by electrical and fiber optic tethers, can be carried by a mouse during ambulatory experiments $^{12}$. Moreover, compact optical implants, such as light-emitting diodes, optical fibers, and integrated photonic waveguides, have been used to deliver or collect photons deep within tissue to record and stimulate disparate regions simultaneously ${ }^{13-20}$. Unlike their external counterparts, these techniques require a physical device to be implanted into the tissue. Therefore, compact and 
flexible devices are highly desired to minimize damage to tissue while still benefiting from the capabilities of optical techniques deep in tissue.

Among different biomedical applications, neurophotonics is an emerging field that demands minimally invasive and highly flexible optical implants for light delivery into the brain with high spatial resolution for optogenetic stimulation and functional imaging of brain activity. To study and understand the distributed and dynamic neural circuits in the brain, advanced methods are needed to monitor and manipulate neuronal activity at single-cell resolution over different areas of the brain during naturalistic behavior.

Brain tissue is especially vulnerable to damage from rigid implants. It has been shown that the performance of neural implants gradually degrades over time due to the foreign body response $(\mathrm{FBR})^{21}$. This biological tissue response, which involves inflammation and astroglial scarring, is believed to be triggered, in part, by the mismatch between the mechanical properties of the implanted device and neural tissue ${ }^{22}$. The buildup of scar tissue around the implantation site degrades the recording signal-to-noise ratio and stimulation efficiency, limiting the lifetime of such implants. For electrical recording, flexible polymer devices have been shown to reduce damage to the brain tissue and thus enable longer-term neural recording ${ }^{23}$. An equivalent flexible optical platform is desired to enable chronic optical interrogation of neural circuits.

Most existing integrated photonic waveguides are made of rigid semiconductor materials and dielectrics such as silicon, silicon nitride, and silicon dioxide ${ }^{24}$. These integrated photonic platforms are mainly designed for optical communications and are not necessarily optimized for implantable or wearable biophotonics. In addition to microfabricated integrated photonic devices, comparatively large single-channel light guides, including optical fibers and polymer silicone light guides ${ }^{25,26}$, have been used for light delivery into tissue. Polymers such as SU-8 have also been incorporated into integrated photonic devices ${ }^{15,27,28}$.

The overall stiffness of a device is determined by its geometry and Young's (elastic) modulus. Therefore, in addition to the material properties, the chronic tissue response is also a function of the shape of the implanted device. Typically, implantable neural probe architectures are fabricated in long, high-aspect-ratio shapes that minimize the cross-sectional area to reduce acute tissue damage during implantation ${ }^{29}$. The probe mechanics in this shape are well characterized by the cantilever stiffness, which scales linearly with the Young's modulus of the material ${ }^{21}$. Compared with other commonly used materials for photonic waveguides, Parylene $\mathrm{C}$ and polydimethylsiloxane (PDMS) exhibit orders of magnitude
Table 1 Mechanical properties of tissues and biomaterials

\begin{tabular}{ll}
\hline Material & Young's modulus, $E(G P a)$ \\
\hline Silicon $^{58}$ & $130-170$ \\
Silicon nitride $^{59}$ & $280-290$ \\
Parylene C $^{60}$ & $1.5-4$ \\
PDMS $^{61}$ & $(1.32-2.97) \times 10^{-3}$ \\
Brain tissue $^{62}$ & $(0.948-3.141) \times 10^{-6}$ \\
Skin $^{63}$ & $(6-222) \times 10^{-6}$ \\
Muscle $^{63}$ & $(2-12) \times 10^{-6}$ \\
\hline
\end{tabular}

lower Young's moduli, closer to the values of most biological tissues (Table 1), suggesting that this architecture will be less damaging to the surrounding tissue after implantation. Although polymer materials are still many orders of magnitude stiffer than the surrounding tissue, histology studies have shown that polymer probes cause a reduced FBR compared with more rigid silicon probes ${ }^{30}$.

Here, we demonstrate an integrated photonic platform to realize compact, biocompatible, and fully flexible polymer-based optical waveguide arrays that can deliver light efficiently into tissue in a minimally invasive way. Our architecture, Parylene photonics, is realized entirely in a flexible, biocompatible material platform composed of Parylene C and PDMS polymers. PDMS is optically transparent in the visible range and is resistant to degradation from prolonged exposure to a biological environment $^{31,32}$. Both polymers are used in FDA-approved medical implants ${ }^{33,34}$ and are also widely used in research as well as clinical applications ${ }^{35}$. With a proven history of biosafety in humans, Parylene $\mathrm{C}$ and PDMS form a compelling photonic platform for biointerface development, with translational potential for medical applications.

\section{Device design and architecture}

\section{Parylene photonic waveguides confine and guide light}

Our flexible photonic platform, Parylene photonics, utilizes a dense array of waveguides in the shape of an implantable probe to deliver light from external light sources deep into tissue (Fig. 1a). Light is coupled to each waveguide from light sources located at the backend of the probe, which can remain outside of the tissue. These light sources can be either integrated laser diodes or a fiber tether connected to an external laser source. The probe has a long flexible shank that is implanted into the tissue to deliver light at the target depth. Here, we have demonstrated $5 \mathrm{~cm}$ long waveguides. To minimize damage to the surrounding tissue, the shank is designed to be very thin ( $7 \mu \mathrm{m}$ total thickness) and narrow $(60 \mu \mathrm{m}$ 
a
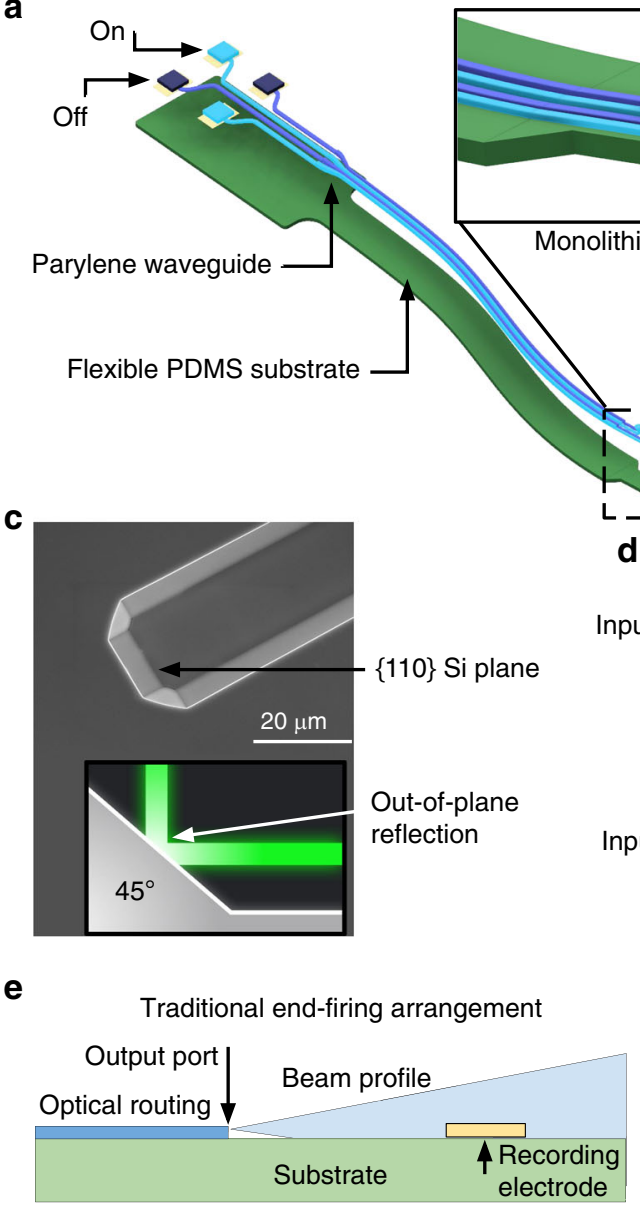

e

C

Traditional end-firing arrangement

Recording electrode b

Waveguide cross-section on wafer

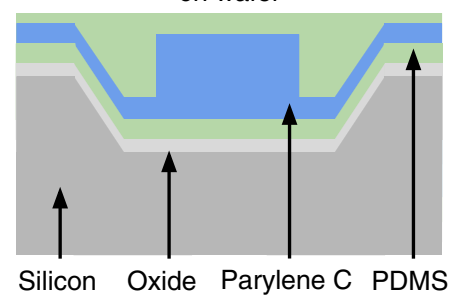

Silicon Oxide Parylene C PDMS

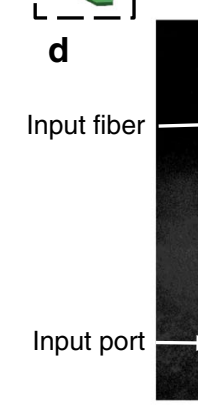

f

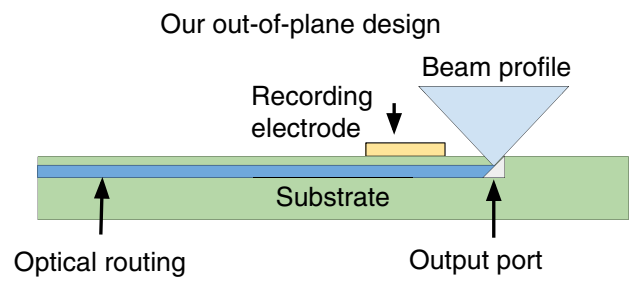

Fig. 1 A conceptual schematic of a Parylene photonic waveguide neural probe showing device features and a comparison with traditional architectures. a Schematic diagram of the Parylene C/PDMS optical waveguide neural probe. The inset image shows the integrated device capable of out-of-plane light delivery. b Schematic of the waveguide transverse cross-section on the wafer. c Scanning electron microscope (SEM) micrograph of a Si trench etched using $\mathrm{KOH}$ with the $\{110\}$ plane indicated, which acts as the mold for the micromirror port, as shown in the crosssectional schematic diagram. d Out-of-plane input coupling from an optical fiber into a Parylene $\mathrm{C}$ waveguide using the $45^{\circ}$ micromirror at the input port. Adjacent waveguides appear bright due to the brightfield illumination and reflections from the bright beam spot of the input fiber. Only a single waveguide is excited, as seen by the bright line of outscattered light in the center of the array. Fiber alignment was performed using a precision micromanipulator (PatchStar, Scientifica, UK). e Schematic of the traditional in-plane illumination from an end-firing waveguide, where waveguide illumination is along the probe shank, limiting the spatial resolution. $\mathbf{f}$ Schematic of the out-of-plane illumination in our design, where the waveguide output is oriented perpendicular to the probe shank, allowing for higher spatial resolution

waveguide pitch). The total shank width is determined by the number of waveguide channels and the size and pitch of waveguides.

Our waveguide core is made of Parylene $C$, a highrefractive-index biocompatible polymer $(n=1.639)$, which is transparent throughout the visible range of the optical spectrum ${ }^{36}$. PDMS is used as the waveguide cladding due to its lower refractive index than that of Parylene C $(n=1.4)$. The material choice of Parylene C and PDMS provides a large index contrast $(\Delta n=0.239)$ among biocompatible polymers to confine an optical mode. A large index contrast improves mode confinement and results in a small bend loss. However, a large index contrast can also exacerbate the scattering losses due to sidewall roughness, which can be alleviated by smoothing the waveguide sidewalls. A cross-sectional schematic of the waveguide structure on the wafer is shown in Fig. 1b.

\section{Micromirrors enable vertical input/output coupling}

An important feature of our Parylene photonics implementation is the monolithic integration of embedded $45^{\circ}$ micromirrors at the input and output ports, which enables broadband input/output coupling of light. The mirror topography is first precisely defined in a silicon 


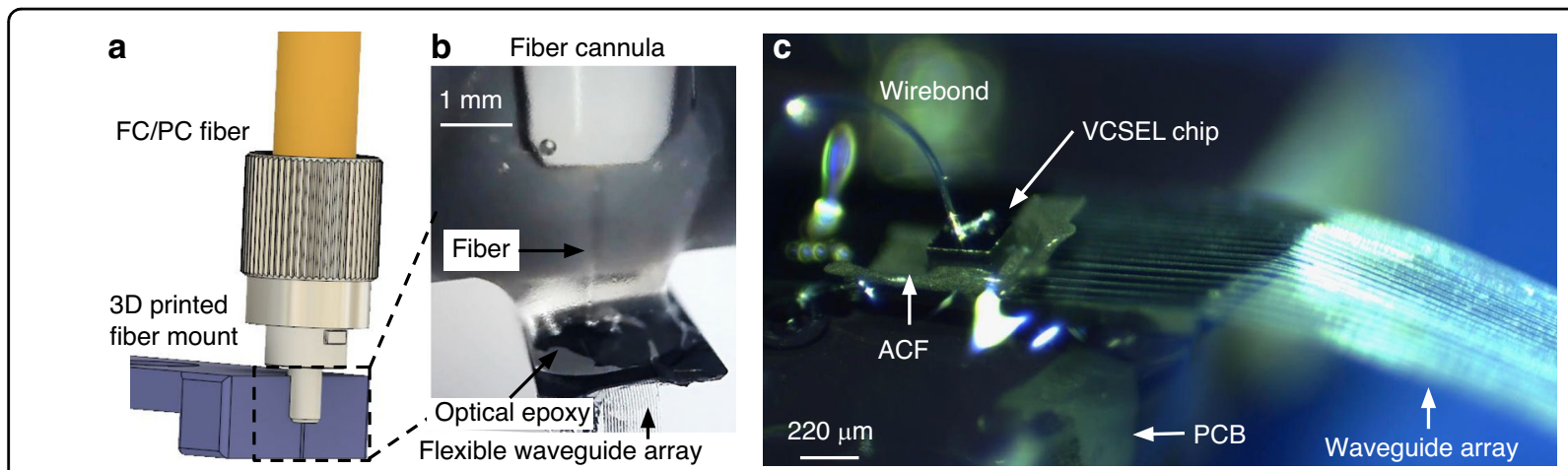

Fig. 2 Packaging of Parylene photonic waveguides with optical fibers and laser diode chips. a Schematic of a fiber-bonding 3D printed mount featuring a V-groove for fiber alignment. b Fiber optic bonded to a flexible Parylene waveguide using optical epoxy. c A vertical-cavity surfaceemitting laser (VCSEL) chip bonded to the input port of a flexible Parylene waveguide using anisotropic conductive film (ACF)

mold (Fig. 1c) and then transferred to the flexible polymer device via deposition of Parylene $\mathrm{C}$ and PDMS onto the $\mathrm{Si}$ mold. These monolithically embedded micromirror structures are capable of $90^{\circ}$ out-of-plane input/output light coupling (Fig. 1d).

As output ports, these micromirrors enable out-ofplane illumination normal to the surface of the implantable probe. Traditional optical waveguides and fibers operate in an end-firing configuration (Fig. 1e), in which light is emitted from the end facet. End-firing waveguides result in an in-plane beam profile that is oriented along the axis of the probe, causing a large portion of the probe surface area to be illuminated and limiting the number of nonoverlapping output ports that can be arranged on the surface. To enable a high spatial resolution illumination pattern along the probe shank, an out-of-plane scheme is preferred (Fig. 1f). In addition, in the context of neural probes, where electrical recording sites are also patterned on the surface of the shank, an out-of-plane beam profile avoids direct illumination of recording electrode sites, reducing the severity of photoelectric artifacts ${ }^{37}$. A comparison of in-plane and out-of-plane illumination profiles is presented in Fig. 1e, f. In-plane mirrors have also been used for side-firing waveguides in order to increase the optical probe spatial resolution ${ }^{38}$, but out-of-plane mirrors are required to collocate stimulation with surface electrode arrays on the same probe shank.

Output ports may be lithographically defined in any desired arrangement along the probe shank to suit the purpose of the intended experiment. Although Parylene photonics can be broadly used in any biomedical application, here, we focus on device design in the context of neural stimulation using optogenetics. For example, output ports may be spaced along the length of the probe shank to stimulate different regions of tissue (e.g., different layers of cortex) or placed in a dense grid for interrogation of neural circuits in the same region.
Packaging with optical fibers allows broadband operation

Packaging of microfabricated optical waveguides with light sources is a uniquely stringent requirement for implantable applications. The device backend must be compact and robust to enable implantation. The embedded micromirror input ports facilitate coupling of light from the surface into our integrated photonic waveguides.

Optical fibers can be aligned to the waveguide input facet using a 3D printed V-groove (Fig. 2a) and directly bonded to the waveguide array with optical epoxy, as shown in Fig. 2b ("Materials and methods"). Due to the compact size of optical fibers $(3.0 \mu \mathrm{m}$ core diameter, $125 \mu \mathrm{m}$ cladding diameter), many fibers may be bonded to the probe backend, allowing independent light coupling to multiple waveguides in the array. The chosen optical fiber (S405-XP, Thorlabs Inc., USA) operates as a singlemode fiber over wavelengths of $400-680 \mathrm{~nm}$, covering the entire visible spectrum, relevant for most optical reporters and commonly used opsins for optogenetic stimulation. Packaging optical fibers at the backend of our implantable optical waveguide arrays has the advantage of enabling operation at different wavelengths using different external laser sources.

\section{Bonding laser diode chips enables compact backends}

It is highly desired that implantable photonic waveguide probes are realized such that the prohibitive tether connections to the backend are either eliminated or at least reduced in size to enable chronic experiments on freeroaming animals. Utilizing the micromirrors for input coupling, compact vertical-cavity surface-emitting laser (VCSEL) chips may be directly bonded to the input facet using a thin layer of anisotropic conductive film (ACF) ("Material and methods"). The resulting probe is shown in Fig. 2c. The diode chips (I0-0680M-0000-A002, Vixar Inc., USA) emit at a wavelength of $\lambda=680 \mathrm{~nm}$ and are both compact $(220 \mu \mathrm{m} \times 220 \mu \mathrm{m})$ and lightweight 
a

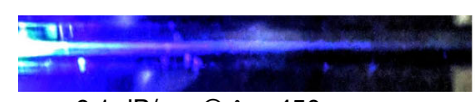

$6.1 \mathrm{~dB} / \mathrm{cm} @ \lambda=450 \mathrm{~nm}$
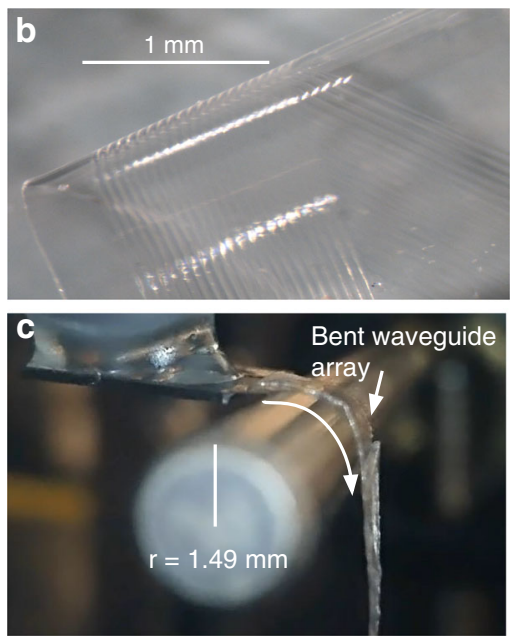

Outscattered light

$4.9 \mathrm{~dB} / \mathrm{cm} @ \lambda=532 \mathrm{~nm}$

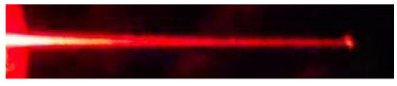

$4.1 \mathrm{~dB} / \mathrm{cm} @ \lambda=633 \mathrm{~nm}$

d Bend loss $(\lambda=633 \mathrm{~nm})$

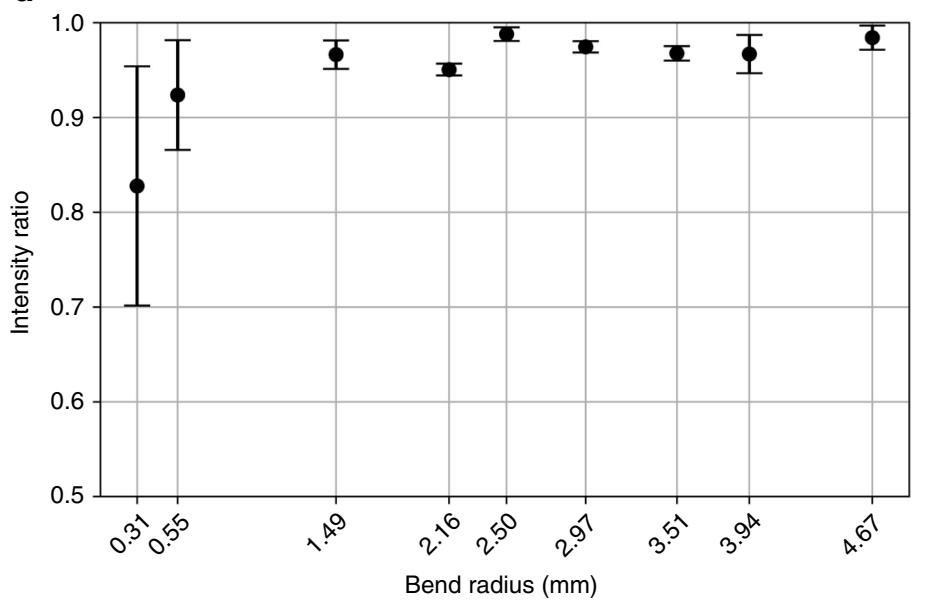

Fig. 3 Demonstration of low propagation loss and bend loss. a Outscattered light along the length of the waveguide, imaged at three different optical wavelengths in the visible range to show the trajectory of guided light. The waveguides were imaged from the side to avoid direct illumination from the output port, and the input power from the laser was increased so that the outscattered light along the waveguide path was clearly visible. $\mathbf{b}$ Micrograph of the released Parylene C/PDMS waveguide array. $\mathbf{c}$ The flexible waveguide array, bent over a custom jig to measure the bend loss (radius $=1.49 \mathrm{~mm}$ ). $\mathbf{d}$ Waveguide bend loss measurements of the relative output intensity (normalized to the output intensity from a straight waveguide) through a $90^{\circ}$ bend of various radii. Low waveguide bend losses are demonstrated with a high intensity ratio (more than 95\%) at millimeter-scale bends. The number of measurements for each datapoint was $n=4$. The error bars denote the standard deviation

$(0.5 \mathrm{mg})$. The ACF provides mechanical and electrical connections to the VCSELs without significantly attenuating the light. We measured an optical transmission of more than $67 \%$ through the ACF across the visible range of optical wavelengths. Direct integration of light sources obviates the need for an external fiber-coupled laser source. Thus, our optical probe requires only an external electrical connection to a pair of small wires, which can be much less cumbersome and restrictive than a brittle, delicate fiber connection.

\section{Results}

\section{Parylene photonic waveguides guide light with low propagation loss}

The Parylene photonics platform operates over a wide range of wavelengths, especially in the visible range that is relevant for optogenetic stimulation of neural activity. The input/output mirrors are broadband and enable coupling of light at different wavelengths (Fig. 3a). The propagation losses were measured at different wavelengths of interest for optogenetic stimulation including $450 \mathrm{~nm}$ (ChR2), $532 \mathrm{~nm}$ (Arch), and 633 and $680 \mathrm{~nm}$ (redshifted opsins, e.g., Chrimson) ${ }^{39}$. Table 2 lists the measured propagation losses in comparison with other waveguide technologies at different wavelengths. Measurement uncertainty is reported for the waveguide-to- waveguide measurement variation. These results show that the propagation losses of our Parylene $C$ waveguides are comparable with those measured in two different $\mathrm{SiN}$ waveguides used in neural probes (Table 2). The specific propagation loss of a microfabricated waveguide depends on its geometry and material properties as well as the fabrication process optimization. Our previous work showed that the primary source of optical loss is the waveguide sidewall roughness as a result of etching the outline of the waveguide core ${ }^{40}$. Parylene photonic waveguides exhibit low losses over a wide range of the spectrum, including $450-680 \mathrm{~nm}$, and the input/output coupling is broadband. Therefore, Parylene photonics can operate over a wide range of wavelengths.

\section{Flexible Parylene waveguides can guide light with low bend loss}

The Parylene photonics platform is designed as a flexible photonic architecture that can freely flex with the tissue to avoid exerting strain on the tissue (Fig. 3b). To operate reliably in vivo, the bends induced by tissue motion should not significantly impact the delivered optical power at the output port. However, bending of optical waveguides results in radiation of confined optical modes before light reaches the output facet. To characterize the overall bend loss, we experimentally 
Table 2 Comparison of neural probe waveguide propagation losses ${ }^{a}$

\begin{tabular}{lllll}
\hline Work & Material platform & Wavelength $\boldsymbol{\lambda}(\mathbf{n m})$ & Propagation loss $\boldsymbol{a}(\mathbf{d B} / \mathbf{c m})$ & $\boldsymbol{n}$ (number of waveguides measured) \\
\hline This work & Parylene C & 450 & $6.1 \pm 1.4$ & 3 \\
Zorzos et al. ${ }^{64}$ & SiN & 473 & 3.2 & 12 \\
This work & Parylene C & 532 & $4.9 \pm 1.2$ & 14 \\
This work & Parylene C & 633 & $4.1 \pm 0.8$ & \\
Kampasi et al. ${ }^{65}$ & SiN & 635 & 5.0 & 5 \\
This work & Parylene C & 680 & $3.2 \pm 1.7$ & \\
\hline
\end{tabular}

${ }^{\text {a }}$ Measurement uncertainty is reported as \pm 1 standard deviation

measured the bend losses at different radii of curvature using a custom-designed jig (Fig. 3c). This jig has two parts. The first part is a 3D printed V-groove to hold the input fiber in place, and the second part is a precisely machined cylindrical rod to form the bend geometry. We used a series of different rods with different diameters to study the effect of bend loss at different bending radii.

The experimental bend loss measurement results are presented in Fig. 3d, showing negligible bend losses for millimeter-scale bends, i.e., more than $95 \%$ of the maximum output intensity even at a bend radius of $1.5 \mathrm{~mm}$. For bend radii smaller than $1 \mathrm{~mm}$, the variance in the measurements is larger than that in the measurements at larger radii of curvature. This variance is caused by experimental challenges in wrapping the probe smoothly around rods at such small radii. We suspect that loops or creases formed in the probe shank during these tight bends reduce the measurement accuracy. Overall, the data suggest that the presented flexible waveguides preserve their performance through millimeter-scale bends in the probe shank. Bends of this size $(1.5-5 \mathrm{~mm})$ are likely to occur during implantation and routing of the flexible shank in the body. Our bend loss measurement results suggest that the output optical power will be minimally affected by flexing in the tissue after implantation.

\section{Embedded micromirrors enable out-of-plane illumination with localized beam profiles}

In addition to enabling broadband vertical input coupling, the $45^{\circ}$ output micromirrors are capable of localized broadband illumination normal to the probe surface. To characterize the output beam profile, a charge-coupled device (CCD) camera was aligned to the output port of a Parylene waveguide in an array, and input coupling was adjusted to maximize the light intensity at the output port (Fig. 4a). Subsequently, to image the beam profile, a block of fluorescent tissue phantom $(0.6 \%$ agar mixed with $10 \mathrm{ppm}$ AlexaFluor 532) was aligned above the output port with a micromanipulator and imaged from the side (Fig. 4b). The resulting fluorescent emission profile is shown in Fig. 4c, with isointensity contours superimposed on the image to show the spatial decay of the light intensity.

The output beam profile reflected by the micromirror was quantitatively measured by imaging the output light intensity at multiple angles (Fig. 4d). Characterization of a $30 \mu \mathrm{m} \times 5 \mu \mathrm{m}$ micromirror output port at $\lambda=532 \mathrm{~nm}$ shows a directional beam profile $\left(1 / \mathrm{e}^{2}\right.$ beam width is $13^{\circ}$ orthogonal to the surface of the probe (Fig. 4e, f). This localized illumination profile allows for multiple output ports to be independently spaced along the probe surface for targeted light delivery. The light intensity directly reflected from the output micromirror port was measured via a CCD camera to be $>36 \mathrm{~dB}$ more intense than the outscattered light from the waveguide.

\section{Design of the cladding thickness enables integration of functional electrical layers}

In the context of neural interfaces, both electrical recording and optical stimulation capabilities are desired to enable simultaneous electrophysiology recording and optogenetic stimulation experiments in the brain. Recording electrodes are usually formed via exposed metal sites connected by traces embedded in polymer insulation $^{41,42}$. A conceptual schematic diagram of an additional planar layer of recording electrodes on a Parylene photonic waveguide is shown in Fig. 5a.

One concern of combining electrical and optical functionalities on the same platform is the interaction of the optical waveguide modes with electrical traces, which will decrease the delivered optical power due to absorption losses in the metal. Commonly used metals such as $\mathrm{Au}, \mathrm{Pt}$, $\mathrm{Ti}$, and $\mathrm{Al}$ exhibit large absorption coefficients in the visible range of the optical spectrum ${ }^{43}$. In our device architecture, electrical traces can be routed along the length of the device, parallel to the optical waveguides. Therefore, any significant interaction between the guided optical mode and metal traces would cause significant attenuation of light after traversing the full length of the device. This interaction can be minimized by routing the electrical traces through a separate layer, vertically spaced from the photonic layer by the PDMS cladding (Fig. 5b). 


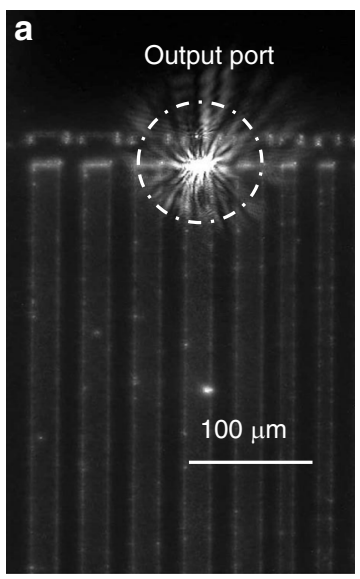

b

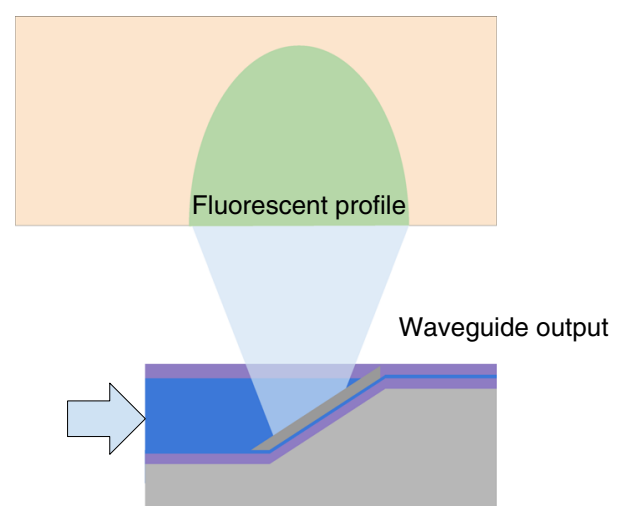

f

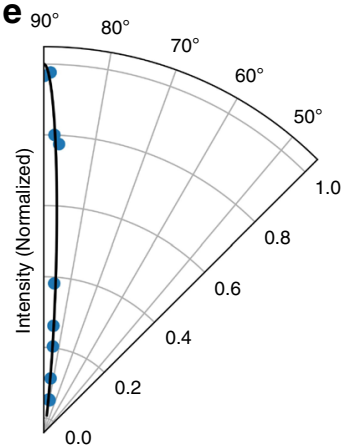

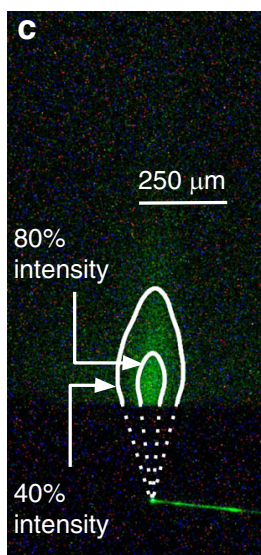

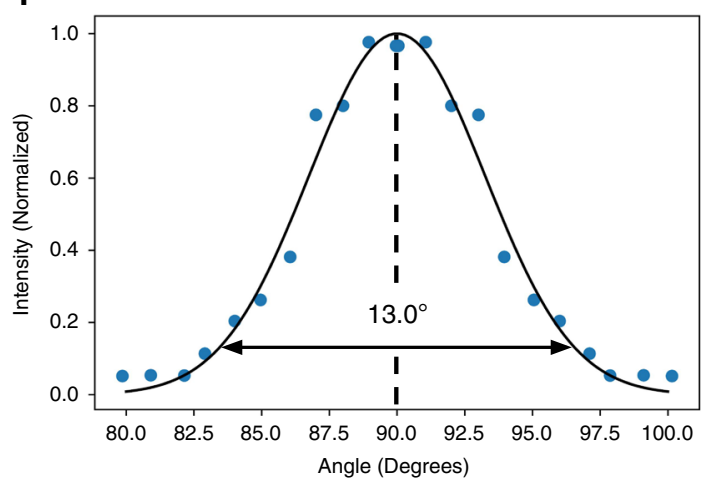

Fig. 4 Directional out-of-plane illumination using output micromirrors. a A brightfield image of the waveguide array (top view), featuring an illuminated output port. b Schematic illustration of the fluorescent beam imaging experimental setup. c Out-of-plane beam profile imaged in a fluorescent tissue phantom with labeled isointensity contours. $\mathbf{d}$ Schematic of the beam profile characterization system. e Radial beam profile, showing a peak intensity at $90^{\circ}$, with rapid off-axis decay. $\mathbf{f}$ Gaussian curve fit to the radial beam profile, showing a beam divergence of $13^{\circ}\left(1 / \mathrm{e}^{2}\right.$ beam width)

To study the optical propagation loss due to the electrical traces, we performed rigorous finite difference eigenmode (FDE) simulations of the waveguide geometry $(30 \mu \mathrm{m} \times$ $5 \mu \mathrm{m})$ with a thin sheet of metal $(200 \mathrm{~nm}$ of $\mathrm{Pt})$ situated over the cladding.

The Parylene photonic waveguide with dimensions of $30 \mu \mathrm{m} \times 5 \mu \mathrm{m}$ is multimode in the visible range. Since each mode profile has a different spread outside and around the waveguide core, the modes experience different levels of attenuation from the presence of the metal sheet. However, when such a multimode waveguide is excited by an external light source (i.e., a laser), optical power is preferentially coupled to lower order modes due to the larger overlap between the typical Gaussian mode profile of the light source and the optical mode profiles of the lower order modes. Optical losses caused by interactions with the Pt layer were modeled for the lowest 30 modes and were found to be less than $3 \times 10^{-10} \mathrm{~dB} / \mathrm{cm}$ for the fundamental mode and less than $5 \times 10^{-8} \mathrm{~dB} / \mathrm{cm}$ for each of the remaining simulated modes (Fig. 5c). These results demonstrate that $1 \mu \mathrm{m}$ of PDMS cladding is sufficiently thick to insulate the waveguide modes from interaction with additional metal layers.

\section{Discussion}

The embedded micromirror input/output port is a versatile feature of our platform. The micromirrors are broadband, unlike traditional out-of-plane illumination mechanisms such as grating couplers, which are highly wavelength dependent. Using the micromirrors, light at multiple wavelengths can be coupled to the waveguides. In the context of neural probes, different optogenetic wavelengths can be used to switch between stimulation and inhibition or to perform cell-type specific targeting. This Parylene photonics platform is the first to enable such high-resolution, broadband, out-of-plane light delivery in a fully compliant and biocompatible platform.

The waveguide output power can be controlled by changing the input optical power. Off-target illumination is minimal since the extinction between the output port 


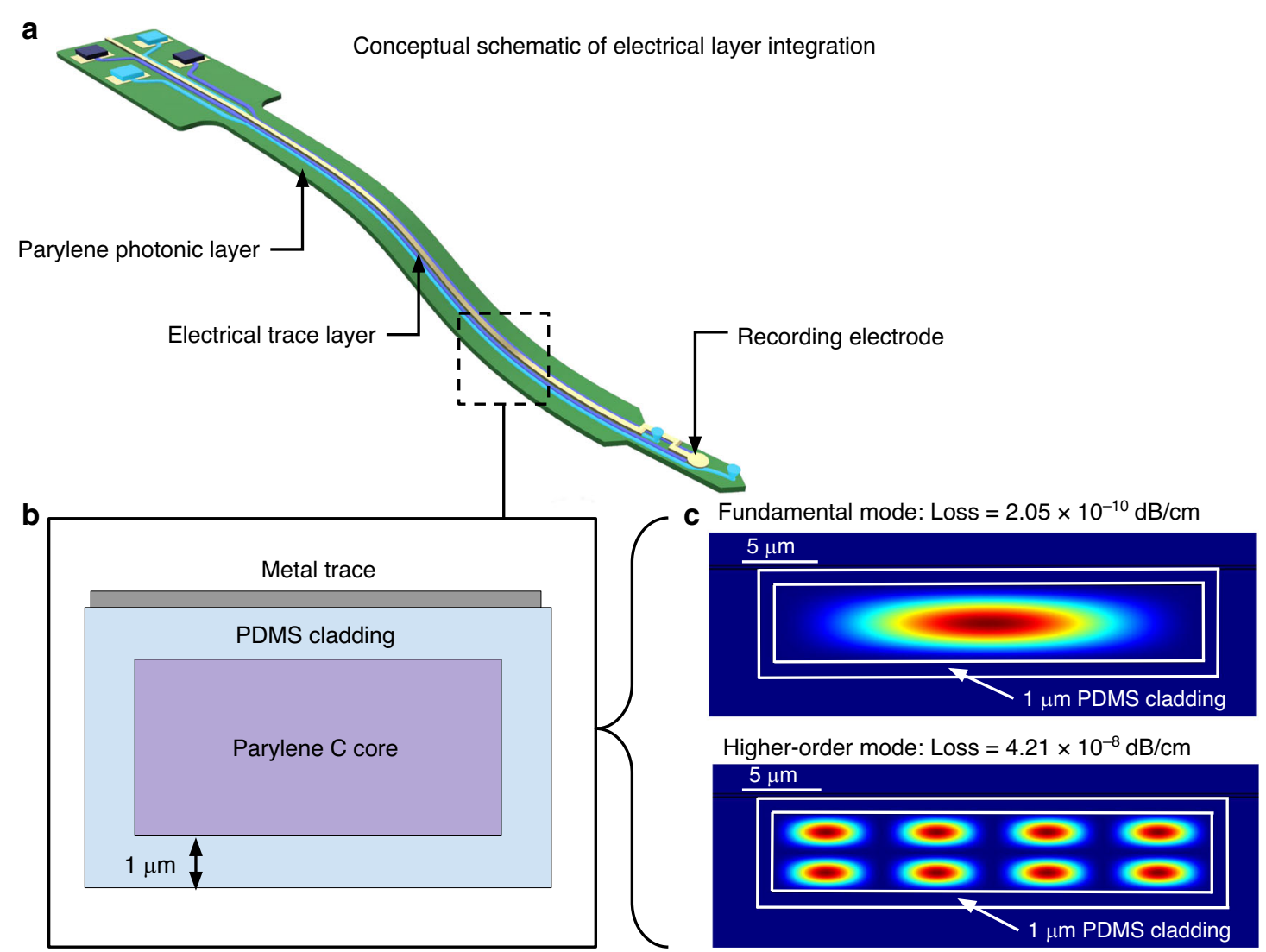

Fig. 5 Feasibility of integrating recording electrodes with Parylene photonic waveguides. a Schematic of a Parylene photonic neural probe with an additional layer of recording electrodes. $\mathbf{b}$ Schematic cross-section of the device shank, with a metal trace over the waveguide core on top of the cladding. c Modal analysis of the waveguide in the presence of the metal traces. Here, due to minimal interaction with the metal layer, both the fundamental mode and higher-order modes show negligible losses

light intensity and the outscattered background light from the probe shank near the output port is $>36 \mathrm{~dB}$. If the input power is very high, outscattered light along the probe shank can be further reduced by using additional optical shielding layers. For biological experiments, the input power must be carefully chosen to achieve an output power that is higher than the threshold of activation or detection of the optical agent of interest while also remaining lower than the threshold of photothermal damage to the tissue at the wavelength of operation.

The Parylene photonics platform utilizes flexible polymer materials to reduce the tissue response after implantation. However, the overall device stiffness depends on the shape of the probe cross-section in addition to the material platform. For example, wide and thin probes are highly compliant when bent along the probe length but have higher stiffness along the width of the probe, resulting in a greater tissue response along the probe edges ${ }^{44}$. Therefore, a neural probe design with a compact footprint (i.e., thin and narrow), in addition to a soft material platform, is necessary to minimize damage to the tissue. When designing a specific implant using Parylene photonics, the number and size of optical channels must be chosen such that the overall width of the probe is minimized.

Here, we report relatively large waveguides $(30 \mu \mathrm{m} \times$ $5 \mu \mathrm{m})$ for a proof-of-principle demonstration. However, optical mode simulations show that the refractive index contrast between Parylene C and PDMS is sufficiently large to realize ultracompact optical waveguides that have well-confined modes even for small cross-sectional dimensions of $1 \mu \mathrm{m} \times 1 \mu \mathrm{m}$. At this small size, the mode exposure to the sidewall is increased, which necessitates process optimization to fabricate such devices with smooth sidewalls and reduce scattering losses. In a dense array configuration $(1 \mu \mathrm{m}$ gap), such waveguides exhibit negligible crosstalk of less than $-30 \mathrm{~dB}$ over $5 \mathrm{~cm}$ length (Supplementary Material, Appendix I). These simulations suggest that under ideal conditions, the presented platform can be scaled to realize waveguide arrays even with an extremely dense pitch of $2 \mu \mathrm{m}$.

Another factor that limits the size and density of a multichannel device is the light source coupling at the 
device input. Here, we have demonstrated bonding of a single fiber to the waveguide input facet. Although the fiber core and cladding are small $(125 \mu \mathrm{m}$ diameter), serial bonding of individual fibers must take into account the prohibitive size of the fiber ferrule and its sleeve, which is typically $2.5 \mathrm{~mm}$. Scaling the bonding process to many channels will require matching the waveguide spacing to the pitch of commercially available photonic chip coupler arrays, which are now available at channel pitches in the range of 20-127 $\mu \mathrm{m}$ (PLC Connections, USA).

In addition to coupling light from benchtop laser sources with a fiber tether, we leverage the versatility of the embedded micromirror input ports for direct out-ofplane coupling of light to the polymer waveguides from laser diode chips. The low weight of the VCSEL sources $(0.5 \mathrm{mg} /$ diode $)$ is important in the context of chronic experiments on freely moving subjects, where the weight budget is typically $10 \%$ of the weight of the animal (2-3g, headstage weight limit for mice ${ }^{45}$ ). This integrated laser diode platform may be directly powered and modulated via electrical power supplies integrated into a headstage or used for tetherless experiments with the addition of a battery and radio frequency (RF) module. Due to the relatively large output facet $(65 \mu \mathrm{m})$ of the bare chip VCSEL sources used here, a large input port is required to achieve efficient coupling into the waveguide. In future designs, the waveguide can be tapered to achieve high coupling efficiency while routing compact waveguides in the probe shank, or more compact laser diodes can be used.

The fabrication process outlined in this paper to realize Parylene photonics is compatible with commonly used microfabrication techniques. During the fabrication process, harsh chemicals such as hydrofluoric (HF) acid are employed as an efficient way to remove the oxide hardmask and sacrificial layers, necessitating careful rinsing in deionized water to avoid tissue contamination. Other hardmask and sacrificial release layers, such as germanium ${ }^{46}$, which can be removed using biosafe solvents such as $1 \%$ hydrogen peroxide, could be explored as alternatives. Our scalable microfabrication process enables monolithic integration of additional planar structures prior to release. Thus, using this platform, additional photonic layers can be stacked to increase device density, or electrical layers can be added to create a multimodal flexible device platform.

Although flexible devices are less damaging during chronic experiments, they are difficult to implant because they lack the structural rigidity needed to penetrate tissue. To address this need, implantation cannulas and bioresorbable stiffeners have been developed to temporarily increase the stiffness of the device for implantation. Bioresorbable materials such as polyethylene glycol, silk, callagen/gelatin, and PLGA have demonstrated tunable properties for both the stiffness and dissolution rate, which may be explored in combination with Parylene photonics ${ }^{47}$. The required insertion force can be reduced by shaping the bioresorbable stiffener into a sharp tip using controlled dip coating ${ }^{48}$ or a molding process ${ }^{49}$. In addition, external braces may be added to increase the buckling force of the implantable device ${ }^{50}$. These parameters require additional optimization of the Parylene photonics architecture, but compatibility with existing techniques allows future implementations to benefit from the rich literature on implantation techniques for flexible neural probes.

\section{Conclusion}

Parylene photonics shows great promise for realizing flexible chronic implantable biointerfaces, including neural probes, which can reduce the FBR in tissue. The out-of-plane, broadband input/output ports enabled by embedded micromirrors allow devices to create patterns of localized illumination beams normal to the surface for collocated integration with recording electrodes and enable direct packaging with light sources on the probe backend. This photonic device platform is broadband and offers unprecedented flexibility in choosing the desired wavelength of light for opsins and optical reporters. While in this paper, we discussed packaging and implantation considerations to show the feasibility of using this platform to realize optical neural probes, Parylene photonics can be used in many other biomedical applications where a flexible, biocompatible optical device is desired.

\section{Materials and methods \\ Fabrication process \\ Preparing the silicon mold}

To implement Parylene $\mathrm{C}$ photonic waveguides and integrated micromirrors, fabrication was performed on 4inch silicon wafers $(n\{100\})$ with $1 \mu \mathrm{m}$ thermal oxide. The waveguide fabrication process flow is shown in Fig. 6 (with additional process details in Table 3). The thermal oxide layer serves as a hardmask for deep etching of silicon. Oxide was patterned using optical lithography and anisotropic reactive ion etching (RIE) (Step 1 in Table 3). Then, $45^{\circ}$ Si sidewalls were formed using wet etching in potassium hydroxide $(\mathrm{KOH})$ mixed with Triton X-100 surfactant $^{51}$ (Step 2 in Table 3) to reach the desired trench depth of $6 \mu \mathrm{m}$ (Fig. 6a). The oxide hardmask was subsequently stripped via wet etching in $49 \%$ HF. Careful design of the mask orientation with features at $45^{\circ}$ to the (100) plane, indicated by the wafer main flat, is required to expose the (110) crystal plane and define the micromirror surface $^{51}$. The patterned Si surface serves as a mold for subsequent polymer layers, defining the $3 \mathrm{D}$ shape of the micromirrors. Subsequently, $300 \mathrm{~nm}$ of conformal oxide was deposited on the patterned Si surface using a plasma- 


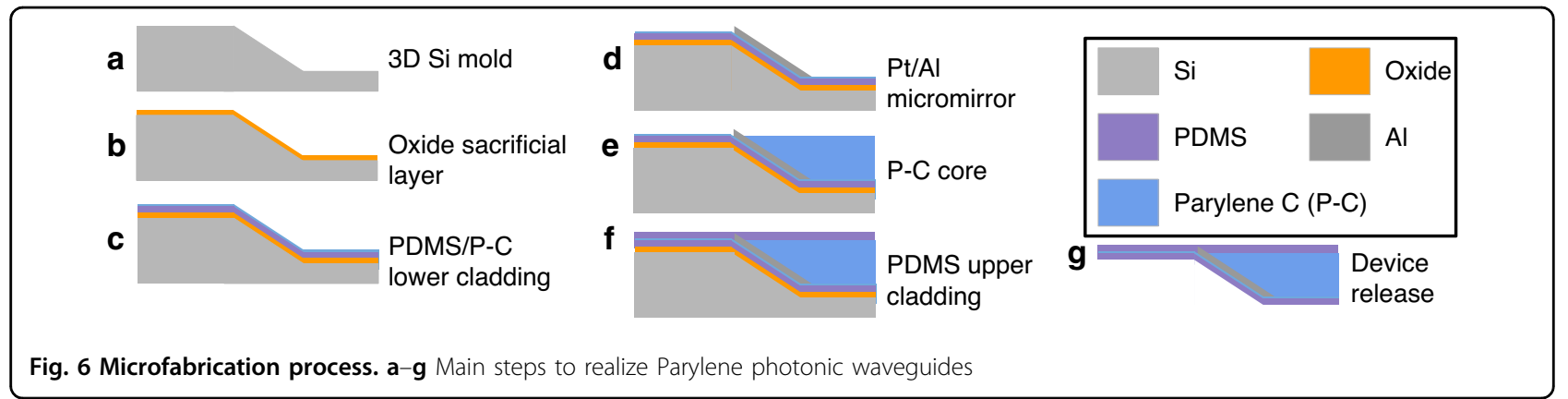

Table 3 Fabrication process parameters

\begin{tabular}{|c|c|c|c|}
\hline Process step & Tool & Parameters & Rate \\
\hline (1) Thermal oxide etch & PlasmaTherm 790 RIE & $\begin{array}{l}\text { Gas: } 22.5 \mathrm{SCCM} \mathrm{CHF}_{3} \\
\text { Gas: } 16 \mathrm{SCCM} \mathrm{O} \\
\text { Pressure: } 100 \mathrm{mTorr} \\
\text { Power: } 200 \mathrm{~W}\end{array}$ & $55 \mathrm{~nm} / \mathrm{min}$ \\
\hline (2) Anisotropic Si etch & Wet Bench & $\begin{array}{l}\text { Concentration: } 2 \mathrm{M} \mathrm{KOH} \\
\text { Concentration: } 60 \mathrm{ppm} \text { Triton X-100 Surfactant } \\
\text { Temperature: } 90^{\circ} \mathrm{C} \\
\text { Agitation: } 210 \mathrm{rpm} \text { stirring }\end{array}$ & $280 \mathrm{~nm} / \mathrm{min}$ \\
\hline (3) PECVD oxide deposition & Trion Orion II PECVD & $\begin{array}{l}\text { Temperature: } 375^{\circ} \mathrm{C} \\
\text { Pressure: } 900 \text { mTorr } \\
\text { Gas: } 75 \mathrm{SCCM} \mathrm{N} \mathrm{SC}_{2} \\
\text { Gas: } 70 \mathrm{SCCM} \mathrm{SiH}_{4}\end{array}$ & $60 \mathrm{~nm} / \mathrm{min}$ \\
\hline (4) Parylene deposition & SCS Labcoter-2 & $\begin{array}{l}\text { Furnace temperature: } 690^{\circ} \mathrm{C} \\
\text { Chamber gauge temperature: } 135^{\circ} \mathrm{C} \\
\text { Vaporizer temperature: } 175^{\circ} \mathrm{C} \\
\text { Pressure: } 35 \text { mTorr }\end{array}$ & $2 \mathrm{~g} \rightarrow 1.47 \mu \mathrm{m}$ \\
\hline (5) Pt evaporation & Kurt J. Lesker PVD 75 Electron Beam Evaporator & Pressure: $3 \times 10^{-7}$ Torr & $3 \AA / s$ \\
\hline (6) Al Evaporation & Kurt J. Lesker PVD 75 Electron Beam Evaporator & Pressure: $3 \times 10^{-7}$ Torr & $5 \AA / s$ \\
\hline (7) Cr sputtering & CVC Connexion Sputtering System & $\begin{array}{l}\text { Pressure: } 7 \text { mTorr } \\
\text { Power: } 50 \text { W RF } \\
\text { Gas: } 50 \text { SCCM Ar }\end{array}$ & $10 \mathrm{~nm} / \mathrm{min}$ \\
\hline (8) Parylene etching & Trion Phantom II RIE & $\begin{array}{l}\text { Gas: } 14.0 \mathrm{SCCM} \mathrm{O} \\
\text { Pressure: } 50 \mathrm{mTorr} \\
\text { Power: } 50 \mathrm{~W} \text { RF }\end{array}$ & $250 \mathrm{~nm} / \mathrm{min}$ \\
\hline (9) Al sputtering & Perkin Elmer $8 \mathrm{~L}$ Sputtering System & $\begin{array}{l}\text { Power: } 100 \mathrm{~W} \text { RF } \\
\text { Pressure: } 5 \mathrm{mT}\end{array}$ & $30 \mathrm{~nm} / \mathrm{min}$ \\
\hline (10) PDMS etching & Trion Phantom II RIE & $\begin{array}{l}\text { Pressure: } 75 \mathrm{mT} \\
\text { Gas: } 30 \mathrm{SCCM} \mathrm{CF} 4 \\
\text { Gas: } 10 \mathrm{SSCM} \mathrm{O}_{2} \\
\text { Power: } 200 \mathrm{~W}\end{array}$ & $200 \mathrm{~nm} / \mathrm{min}$ \\
\hline (11) Si etching & Trion Phantom II RIE & $\begin{array}{l}\text { Pressure: } 25 \text { mTorr } \\
\text { Power: } 100 \mathrm{~W} \\
\text { Gas: } 30 \text { SCCM SF } 6\end{array}$ & $2.3 \mu \mathrm{m} / \mathrm{min}$ \\
\hline
\end{tabular}


enhanced chemical vapor deposition (PECVD) process (Step 3 in Table 3) as a sacrificial layer to enable device release from the Si mold (Fig. 6b).

\section{Spin-coating PDMS substrate}

To form the substrate for the waveguide structure, a $1 \mu \mathrm{m}$ PDMS layer was spin-coated on the silicon mold. Due to the high viscosity of PDMS, such a thin layer requires dilution with hexane prior to spin-coating. PDMS (Sylgard 184, Dow Corning Corp, USA) was diluted to 1:10 PDMS:hexane by volume, thoroughly mixed, and filtered through a $0.2 \mu \mathrm{m}$ membrane filter to remove any particulates. The solution was spin-coated for $60 \mathrm{~s}$ at $2000 \mathrm{rpm}$ and then degassed in $400 \mathrm{~m}$ Torr vacuum for $4 \mathrm{~min}$. Finally, wafers were oven-baked for $45 \mathrm{~min}$ at $100^{\circ} \mathrm{C}$ to cure the thin film and remove the solvent. To verify the thickness of PDMS to be $1 \mu \mathrm{m}$, the thin film was measured using a surface profilometer (P-15 Stylus Profiler, KLA Tencor, USA), wherein PDMS was mechanically removed from a portion of the wafer to measure the step height. The PDMS spin-coating process is not perfectly conformal and is affected by the waveguide trench topography. The spin-coating parameters and size of the trench must be optimized to achieve the desired thickness at the bottom of the trench. Multiple waveguides can be routed through a wide common trench.

\section{Metal micromirrors}

Due to the low surface energy of PDMS, photoresist cannot be directly spin-coated on its surface for lithography $^{52}$. To overcome this issue, we developed and optimized a fabrication process in which a very thin $(300 \mathrm{~nm}$ ) layer of Parylene C film was deposited on PDMS to serve as an adhesion layer for the photoresist and enable optical lithography (Fig. 6c, Step 4 in Table 3). Direct chemical vapor deposition (CVD) of Parylene C on PDMS provides strong adhesion, making Parylene an ideal material ${ }^{53}$. Embedded metal micromirrors were patterned using a lift-off process consisting of lithography (AZ 4210) and evaporation of $5 \mathrm{~nm} \mathrm{Pt}$ and $100 \mathrm{~nm} \mathrm{Al}$ films (Steps 5 and 6 in Table 3). Pt serves as a strong adhesion layer to Parylene $\mathrm{C}^{54}$, while $\mathrm{Al}$ is chosen as the mirror surface for its high reflectance across the visible spectrum $^{55}$. Lift-off was performed via acetone soaking, followed by pulsed (5-10 s) sonication (Fig. 6d). The root mean square surface roughness of the $\mathrm{Al}$ micromirrors was measured via an optical profilometer (Zygo NV7000, AMETEK, USA) to be $49.3 \mathrm{~nm}$ with a standard deviation of $3.7 \mathrm{~nm}$ from mirror to mirror $(n=10)$.

\section{Waveguide core etching and smoothing}

In the next step, the waveguide core was realized in a subsequent layer of Parylene C. We fabricated multimode Parylene photonic waveguides with a core thickness of
$3.5 \mu \mathrm{m}$. Parylene $\mathrm{C}$ was therefore deposited to a thickness of $3.5 \mu \mathrm{m}$ using the CVD process described earlier. To define the outlines of individual waveguides, Parylene $\mathrm{C}$ was removed from the surrounding regions (Fig. 6e) using an anisotropic oxygen plasma etching process. We used a $40 \mathrm{~nm}$ sputtered chromium $(\mathrm{Cr})$ hard mask (Step 7 in Table 3) to achieve a high selectivity for etching Parylene $\mathrm{C}$. The waveguide patterns were aligned to the mirrors using a contact lithography process (AZ 5214E), and the hard mask was patterned by wet etching of $\mathrm{Cr}(\mathrm{Cr} 1020$ Etchant, Microchem GmbH, DE).

The patterns were then transferred to Parylene $C$ via oxygen plasma RIE (Step 8 in Table 3). PDMS acts as an etch stop layer since this polymer is not effectively etched by oxygen plasma alone ${ }^{56}$. After Parylene $C$ was etched (verified via reflectometry), the $\mathrm{Cr}$ hardmask was stripped with $\mathrm{Cr}$ etchant. The etched sidewall roughness results in optical scattering and significant propagation loss, thus rendering the optical waveguide impractical for efficient guiding of light. To address this issue, we used our previously reported technique of depositing an additional $1.3 \mu \mathrm{m}$ conformal layer of Parylene $\mathrm{C}$ over the etched sidewalls to reduce the sidewall roughness and the associated propagation $\operatorname{loss}^{40}$. We use this technique here to smoothen the etched sidewalls and reduce the propagation loss of the implantable waveguides. The three sequential Parylene $C$ layers, i.e., the thin layer on the PDMS substrate, the waveguide layer, and the conformal coating on the top, form the waveguide core with a total thickness of approximately $5 \mu \mathrm{m}$ (Fig. 1b).

\section{Device release}

After the upper cladding of $1 \mu \mathrm{m}$ PDMS was spincoated (Fig. 6f), a $1 \mu \mathrm{m} \mathrm{Al} \mathrm{hardmask} \mathrm{was} \mathrm{sputtered} \mathrm{to}$ define the outline of the entire waveguide array (Step 9 in Table 3). The Al hardmask was lithographically patterned (AZ 4210) and wet etched (Al Etchant Type-A, Microchem $\mathrm{GmbH}$, DE). PDMS cladding was etched, and arrays were singulated using RIE (Step 10 in Table 3). Finally, the Al hardmask was stripped. To release the devices, the silicon substrate was first thinned down to $100 \mu \mathrm{m}$ using backside etching in $\mathrm{SF}_{6}$ (Step 11 in Table 3), and then, the thinned $\mathrm{Si}$ wafer was completely etched in a subsequent etching step in $\mathrm{XeF}_{2}$ (Xactix e2). The sacrificial oxide layer serves to protect the backside of the waveguide array. Once $\mathrm{Si}$ was removed, the sacrificial layer was stripped in buffered HF acid (BHF), resulting in a released, flexible waveguide array (Fig. 6g). The devices were thoroughly rinsed in deionized water after release to avoid contamination of biological tissues by the process chemicals.

\section{Optical fiber bonding}

First, a drop of optical quality epoxy (EPO-TEK 301, Epoxy Technology Inc., USA) was placed on the 
waveguide array backend. An optical fiber was fixed to a custom-designed 3D printed fixture with a $\mathrm{V}$-groove and aligned to the input micromirror using a precision micromanipulator (PatchStar, Scientifica Inc., UK). After maximizing the input coupling efficiency, the epoxy was thermally cured $\left(60^{\circ} \mathrm{C}, 2 \mathrm{~h}\right)$ to provide a stable mechanical connection between the fiber and the waveguide array.

\section{VCSEL light source bonding}

ACF film (CP34531-18AK, Dexerials Corporation, Tokyo, Japan) was placed over the input micromirror. The VCSEL chip was then aligned to the input facet using a commercial flip-chip bonding tool (M9A, BE Semiconductor Industries N.V. (Besi), The Netherlands). Once aligned, the ACF was cured $\left(120^{\circ} \mathrm{C}, 15 \mathrm{~min}\right)$ to fix the VCSEL chip in place. The p-contact and n-contact of the diode were electrically connected to an external PCB using an $\mathrm{Al}$ wirebond (Model 7476D, West Bond, Anaheim, CA, USA).

\section{Characterization of propagation loss}

To characterize the device performance, a single-mode fiber (S405-XP, Thorlabs Inc., USA) or a laser diode (PL 450B, OSRAM GmbH, Germany) was aligned to the input facet of the waveguide while imaging the output port onto a CCD camera. The input coupling was optimized by adjusting the position of the light source using a precision micromanipulator (PatchStar, Scientifica Inc.) to maximize the light emission from the output port. Propagation losses in the waveguide were characterized by measuring the decay in the intensity of outscattered light along the length of the optical waveguide ${ }^{40}$. Traditionally, the modified cutback method is used to measure waveguide loss, which involves recording the output light intensity from multiple waveguides of different lengths and interpolating the loss as a function of length. However, our method based on extracting the propagation loss from the outscattered light allows for measurements of individual waveguide performance in one shot and thus eliminates the errors inherent to the cutback method due to waveguide-to-waveguide variations or changes in the input coupling efficiency from one waveguide to another.

\section{Characterization of bend loss}

A single Parylene photonic probe was sequentially wrapped around rods of different radii $(0.3-5 \mathrm{~mm})$ to measure the bend loss. In all cases, the bend angle was $90^{\circ}$. The transmitted output power was measured by imaging the output port onto a CCD camera. To minimize errors due to variations in input coupling conditions, we optimized and maximized the coupling from the input optical fiber by measuring the transmission of the straight waveguide and then permanently affixed the fiber to the input facet using an epoxy to maintain consistent input coupling. We repeated the experiments four times, each time sweeping the range of bend radii, to ensure the repeatability of the results and characterize the measurement error at different radii.

\section{Characterization of the micromirror output beam profile}

The optical properties of the waveguide platform were characterized to demonstrate broadband, localized, outof-plane illumination capabilities. A single-mode optical fiber (S405-XP, Thorlabs Inc., USA) was aligned vertically to the input mirror for input coupling to the waveguide.

The input coupling efficiency was optimized by adjusting the fiber position using a precision micromanipulator (PatchStar, Scientifica Inc.), while monitoring the peak intensity from the output port on the CCD camera (EO-5012M, Edmund Optics, USA).

Output port beam profiles were imaged by rotating the imaging platform in fixed increments about the axis of the waveguide and imaging the output port light intensity. To increase the dynamic range of the system, the imaging exposure time was scaled for each sample to avoid individual pixel saturation, and the pixel intensity values were then scaled by the exposure times.

\section{Agar phantom preparation}

Powdered bacteriological agar (A5306, Sigma-Aldrich, USA) was mixed by weight with $200 \mathrm{~mL}$ of deionized water in a $500 \mathrm{~mL}$ beaker and stirred at $600 \mathrm{rpm}$ while heating on a hotplate. The top of the beaker was covered with a quartz watch glass to prevent evaporation. Once the solution was boiling, the hotplate was switched off, and the temperature was monitored via a thermocouple until dropping to $60^{\circ} \mathrm{C}$. For fluorescent samples, $10 \mathrm{ppm}$ AlexaFluor 532 was added to the solution and mixed for an additional $5 \mathrm{~min}$. The solution was then poured into a mold and cooled in a refrigerator at $6^{\circ} \mathrm{C}$ for $30 \mathrm{~min}$ before use.

\section{Simulation method}

Optical simulations were performed using commercial software (Lumerical MODE Solutions 2018b, Lumerical Inc., USA). The waveguide mode profiles were solved in two dimensions (2D) by taking a cross-section of the device geometry perpendicular to the axis of propagation ( $z$-axis). The FDE method was used to solve Maxwell's equations in $2 \mathrm{D}$ to find the electric and magnetic field profiles for each mode, as well as the complex propagation constant, $\beta$. The mode profiles were visualized by plotting the normalized electric field intensity, $|E|^{2}$. A perfectly matched layer (PML) boundary condition was used around the simulation domain. In all simulations, a background index of 1.0 was used. Simulations were performed at a wavelength of $\lambda=450 \mathrm{~nm}$.

Parylene waveguides were simulated with dimensions of $1 \mu \mathrm{m} \times 1 \mu \mathrm{m}$ and $30 \mu \mathrm{m} \times 5 \mu \mathrm{m}$. 
To simulate the waveguide structure, Parylene $C$ is defined as a dielectric with refractive index of $n=1.639$, and PDMS is defined as having a refractive index of $n=$ 1.4. The platinum optical properties were taken from Palik $^{57}$.

\section{Acknowledgements}

This material is based upon work supported in part by the National Science Foundation under Grant No. 1926804 and also by the National Institute of Neurological Disorders and Stroke of the National Institutes of Health under Award Number 1RF1NS113303. The authors acknowledge support by the Carnegie Mellon University Ben Cook Presidential Graduate Fellowship (J.W.R.), the Carnegie Mellon University Richard King Mellon Foundation Presidential Fellowship in the Life Sciences (J.W.R.), the Axel Berny Presidential Graduate Fellowship (J.W.R.), Philip and Marsha Dowd (J.W.R.), and the National GEM Consortium (M.L.). The authors acknowledge the support of the Carnegie Mellon Nanotechnology facility.

\section{Author contributions}

J.W.R. designed the devices, performed microfabrication, characterization, modeling, simulation, and prepared manuscript and the figures. M.L. performed characterization, packaging, and prepared the figures. M.C. served as the principal investigator on the project, designed and supervised the research, and prepared the manuscript. All authors reviewed the manuscript.

\section{Conflict of interest}

The authors declare that they have no conflict of interest.

Supplementary information accompanies this paper at https://doi.org/ 10.1038/s41378-020-00186-2.

Received: 4 July 2019 Revised: 1 June 2020 Accepted: 3 June 2020 Published online: 21 September 2020

\section{References}

1. Yang, W. \& Yuste, R. In vivo imaging of neural activity. Nat. Methods 14, 349-359 (2017).

2. Wu, D. et al. Fluorescent chemosensors: the past, present and future. Chem. Soc. Rev. 46, 7105-7123 (2017).

3. Carrasco-Zevallos, O. M. et al. Review of intraoperative optical coherence tomography: technology and applications [Invited]. Biomed. Opt. Express 8 1607 (2017).

4. Riley, R. S. \& Day, E. S. Gold nanoparticle-mediated photothermal therapy: applications and opportunities for multimodal cancer treatment. Wiley Interdiscip. Rev. Nanomed. Nanobiotechnol. 9, e1449 (2017).

5. Kim, J., Kim, J., Jeong, C. \& Kim, W. J. Synergistic nanomedicine by combined gene and photothermal therapy. Adv. Drug Deliv. Rev. 98, 99-112 (2016).

6. Li, Z. et al. Small gold nanorods laden macrophages for enhanced tumor coverage in photothermal therapy. Biomaterials 74, 144-154 (2016).

7. Thompson, A. C., Stoddart, P. R. \& Jansen, E. D. Optical stimulation of neurons. Curr. Mol. Imaging 3, 162-177 (2014).

8. Fenno, L., Yizhar, O. \& Deisseroth, K. The development and application of optogenetics. Annu. Rev. Neurosci. 34, 389-412 (2011).

9. de Freitas, L. F. \& Hamblin, M. R. Proposed mechanisms of photobiomodulation or low-level light therapy. IEEE J. Sel. Top. Quantum Electron. 22, 348-364 (2016).

10. Ntziachristos, V. Going deeper than microscopy: the optical imaging frontier in biology. Nat. Methods 7, 603-614 (2010).

11. Deng, X. \& Gu, M. Penetration depth of single-, two-, and three-photon fluorescence microscopic imaging through human cortex structures: Monte Carlo simulation. Appl. Opt. 42, 3321 (2003).

12. Ziv, Y. \& Ghosh, K. K. Miniature microscopes for large-scale imaging of neuronal activity in freely behaving rodents. Curr. Opin. Neurobiol. 32, 141-147 (2015).

13. $\mathrm{Wu}, \mathrm{F}$. et al. An implantable neural probe with monolithically integrated dielectric waveguide and recording electrodes for optogenetics applications. J. Neural Eng. 10, 056012 (2013).
14. Hoffman, L. et al. High-density optrode-electrode neural probe using SixNy photonics for in vivo optogenetics. In 2015 IEEE International Electron Devices Meeting (IEDM) 29.5.1-29.5.4 (IEEE, 2015). https://doi.org/10.1109/ IEDM.2015.7409795.

15. Schwaerzle, M., Paul, O. \& Ruther, P. Compact silicon-based optrode with integrated laser diode chips, SU-8 waveguides and platinum electrodes for optogenetic applications. J. Micromech. Microeng. 27, 065004 (2017).

16. Oh, G., Chung, E. \& Yun, S. H. Optical fibers for high-resolution in vivo microendoscopic fluorescence imaging. Opt. Fiber Technol. 19, 760-771 (2013).

17. Guo, Q. et al. Multi-channel fiber photometry for population neuronal activity recording. Biomed. Opt. Express 6, 3919 (2015).

18. Reddy, J. W., Kimukin, I., Ahmed, Z., Towe, E. \& Chamanzar, M. High density, double-sided, flexible optoelectrical neural probes with embedded microLEDs. Front. Neurosci. 13, 572 (2019).

19. Chamanzar, M., Denman, D. J., Blanche, T. J. \& Maharbiz, M. M. Ultracompact optoflex neural probes for high-resolution electrophysiology and optogenetic stimulation. In 2015 28th IEEE International Conference on Micro Electro Mechanical Systems (MEMS) 682-685 (IEEE, 2015). https://doi.org/10.1109/ MEMSYS.2015.7051049.

20. Klein, E., Gossler, C., Paul, O. \& Ruther, P. High-density $\mu$ LED-based optical cochlear implant with improved thermomechanical behavior. Front. Neurosci. 12, 659 (2018).

21. Kozai, T. D. Y., Jaquins-Gerstl, A. S., Vazquez, A. L., Michael, A. C. \& Cui, X. T. Brain tissue responses to neural implants impact signal sensitivity and intervention strategies. ACS Chem. Neurosci. 6, 48-67 (2015).

22. Moshayedi, P. et al. The relationship between glial cell mechanosensitivity and foreign body reactions in the central nervous system. Biomaterials $\mathbf{3 5}$, 3919-3925 (2014).

23. Weltman, A. et al. Flexible, penetrating brain probes enabled by advances in polymer microfabrication. Micromachines 7, 180 (2016).

24. Thomson, D. et al. Roadmap on silicon photonics. J. Opt. 18, 073003 (2016).

25. Ersen, A. \& Sahin, M. Polydimethylsiloxane-based optical waveguides for tetherless powering of floating microstimulators. J. Biomed. Opt. 22, 055005 (2017).

26. Rehberger, F. et al. Lichtwellenleiter aus PDMS für biomedizinische Anwendungen. Biomed. Eng. Tech. 59, S1068-S1071 (2014).

27. Kwon, K. Y., Lee, H.-M., Ghovanloo, M., Weber, A. \& Li, W. Design, fabrication, and packaging of an integrated, wirelessly-powered optrode array for optogenetics application. Front. Syst. Neurosci. 9, 1-12 (2015).

28. Son, Y. et al. In vivo optical modulation of neural signals using monolithically integrated two-dimensional neural probe arrays. Sci. Rep. 5, 15466 (2015).

29. Szarowski, D. H. et al. Brain responses to micro-machined silicon devices. Brain Res. 983, 23-35 (2003).

30. Lee, H. C. et al. Histological evaluation of flexible neural implants; flexibility limit for reducing the tissue response? J. Neural Eng. 14, 036026 (2017).

31. Heo, C. et al. A soft, transparent, freely accessible cranial window for chronic imaging and electrophysiology. Sci. Rep. 6, 1-11 (2016).

32. Minev, I. R. et al. Electronic dura mater for long-term multimodal neural interfaces. Science. 347, 159-163 (2015).

33. FDA. 21CFR878.3540 (FDA Code of Federal Regulations, Title 21) (FDA, 2018)

34. Stark, N. Literature review: biological safety of Parylene C. Med. Plast. Biomater 3, 30-35 (1996)

35. Marjanović-Balaban, Ž. \& Jelić, D. Polymeric biomaterials in clinical practice. in Biomaterials in Clinical Practice 101-117 (Springer International Publishing 2018). https://doi.org/10.1007/978-3-319-68025-5_4.

36. Jeong, Y. S., Ratier, B., Moliton, A. \& Guyard, L. UV-visible and infrared characterization of poly(p-xylylene) films for waveguide applications and OLED encapsulation. Synth. Metals. 127, 189-193 (2002).

37. Libbrecht, S. et al. Proximal and distal modulation of neural activity by spatially confined optogenetic activation with an integrated high-density optoelec trode. J. Neurophysiol. 120, 149-161 (2018).

38. Zorzos, A. N., Scholvin, J., Boyden, E. S. \& Fonstad, C. G. Three-dimensional multiwaveguide probe array for light delivery to distributed brain circuits. Opt. Lett. 37, 4841 (2012).

39. Klapoetke, N. C. et al. Independent optical excitation of distinct neural populations. Nat. Methods. 11, 338-346 (2014).

40. Reddy, J. W. \& Chamanzar, M. Low-loss flexible Parylene photonic waveguides for optical implants. Opt. Lett. 43, 4112 (2018).

41. Ahmed, Z., Reddy, J. W., Teichert, T. \& Chamanzar, M. High-density steeltrodes: a novel platform for high resolution recording in primates*. In 2019 9th 
International IEEE/EMBS Conference on Neural Engineering (NER) 835-838 (IEEE, 2019). https://doi.org/10.1109/NER.2019.8716921.

42. Ahmed, Z. et al. Flexible ultra-resolution subdermal EEG probes. In 2018 IEEE Biomedical Circuits and Systems Conference, BioCAS 2018-Proceedings (Institute of Electrical and Electronics Engineers Inc., 2018). https://doi.org/ 10.1109/BIOCAS.2018.8584672.

43. Rakić, A. D., Djurišić, A. B., Elazar, J. M. \& Majewski, M. L. Optical properties of metallic films for vertical-cavity optoelectronic devices. Appl. Opt. 37, 5271 (1998).

44. Nguyen, J. K. et al. Mechanically-compliant intracortical implants reduce theneuroinflammatory response. J. Neural Eng. 11, 056014 (2014).

45. Mendrela, A. E. et al. A high-resolution opto-electrophysiology system with a miniature integrated headstage. IEEE Trans. Biomed. Circuits Syst. 12, 1065-1075 (2018).

46. Kalmykov, A. et al. Organ-on-e-chip: three-dimensional self-rolled biosensor array for electrical interrogations of human electrogenic spheroids. Sci. Adv $\mathbf{5}$ eaax0729 (2019).

47. Lecomte, A., Descamps, E. \& Bergaud, C. A review on mechanical considerations for chronically-implanted neural probes. J. Neural Eng. 15, 031001 (2018).

48. Xiang, Z. et al. Ultra-thin flexible polyimide neural probe embedded in a dissolvable maltose-coated microneedle. J. Micromech. Microeng. 24, 065015 (2014).

49. Pas, J. et al. A bilayered PVAVPLGA-bioresorbable shuttle to improve the implantation of flexible neural probes. J. Neural Eng. 15, 065001 (2018).

50. Shoffstall, A. J. et al. A mosquito inspired strategy to implant microprobes into the brain. Sci. Rep. 8, 122 (2018).

51. Rola, K. P. \& Zubel, I. Triton surfactant as an additive to $\mathrm{KOH}$ silicon etchant. J. Microelectromechanical Syst. 22, 1373-1382 (2013).

52. Chen, W., Lam, R. H. W. \& Fu, J. Photolithographic surface micromachining of polydimethylsiloxane (PDMS). Lab Chip. 12, 391-5 (2012).

53. Lim, A. E.-J. et al. Review of silicon photonics foundry efforts. IEEE J. Sel. Top. Quantum Electron. 20, 405-416 (2014).
54. Kuo, J. T. W. et al. Novel flexible Parylene neural probe with 3D sheath structure for enhancing tissue integration. Lab Chip. 13, 554-561 (2013).

55. Hass, G. \& Waylonis, J. E. Optical constants and reflectance and transmittance of evaporated aluminum in the visible and ultraviolet*. J. Opt. Soc. Am. 51, 719 (1961).

56. Garra, J. et al. Dry etching of polydimethylsiloxane for microfluidic systems. J. Vac. Sci. Technol. A Vac., Surf,, Film. 20, 975-982 (2002).

57. Palik, E. D. Handbook of Optical Constants of Solids. III (Academic Press, 1998).

58. Hopcroft, M. A., Nix, W. D. \& Kenny, T. W. What is the Young's modulus of silicon? J. Microelectromechanical Syst. 19, 229-238 (2010).

59. Khan, A., Philip, J. \& Hess, P. Young's modulus of silicon nitride used in scanning force microscope cantilevers. J. Appl. Phys. 95, 1667-1672 (2004).

60. Hassler, C., von Metzen, R. P., Ruther, P. \& Stieglitz, T. Characterization of Parylene $C$ as an encapsulation material for implanted neural prostheses. J. Biomed. Mater. Res. Part B Appl. Biomater. 93, 266-274 (2010).

61. Johnston, I. D., McCluskey, D. K., Tan, C. K. L. \& Tracey, M. C. Mechanical characterization of bulk Sylgard 184 for microfluidics and microengineering. J. Micromech. Microeng. 24, 035017 (2014).

62. Budday, S. et al. Mechanical properties of gray and white matter brain tissue by indentation. J. Mech. Behav. Biomed. Mater. 46, 318-330 (2015).

63. McKee, C. T., Last, J. A., Russell, P. \& Murphy, C. J. Indentation versus tensile measurements of Young's modulus for soft biological tissues. Tissue Eng. Part B. Rev. 17, 155-64 (2011).

64. Zorzos, A. N., Boyden, E. S. \& Fonstad, C. G. Multiwaveguide implantable probe for light delivery to sets of distributed brain targets. Opt. Lett. 35, 4133 (2010).

65. Kampasi, K. et al. Fiberless multicolor neural optoelectrode for in vivo circuit analysis. Sci. Rep. 6, 30961 (2016). 